\title{
Effects of Dual Blockade of the Renin-Angiotensin System in Diabetic Kidney Disease: A Systematic Review and Meta-Analysis
}

\author{
Jacqueline T. Pham ${ }^{1}$, Brian P. Schmitt ${ }^{1,2}$ and David J. Leehey ${ }^{1,2 *}$ \\ ${ }^{1}$ Edward Hines Jr. Veterans Affairs Hospital, Hines, IL 60141, USA \\ ${ }^{2}$ Loyola University Medical Center, Maywood, IL 60153, USA
}

\begin{abstract}
Objective: There is substantial evidence for a renoprotective effect of inhibitors of the renin-angiotensin system (RAS) in diabetic kidney disease (DKD). However, it is unclear whether dual RAS blockade has additional benefits when compared to monotherapy in this population and whether any benefits outweigh the risks.

Data sources: A systematic review and meta-analysis of English language articles was performed using MEDLINE, EMBASE, CINAHL, and the Cochrane database of systematic reviews.

Study selection: All randomized, controlled trials comparing RAS blockade to monotherapy in patients with overt proteinuria were included.

Data extraction: Articles were reviewed independently by two of the authors using a standardized data collection form including study quality indicators.

Data synthesis: All pooled analyses were based on random-effects models. The primary efficacy outcome measure was the percent reduction in proteinuria with combination therapy versus monotherapy measured by difference in means. Secondary outcomes included changes in systolic blood pressure (SBP), glomerular filtration rate (GFR), and serum potassium, and incidence of hyperkalemia. The primary safety outcome was hyperkalemia.

Results: Compared to monotherapy, combination therapy with an angiotensin converting enzyme inhibitor (ACEI) plus angiotensin receptor blocker (ARB) reduced proteinuria by an additional $25 \%$ (mean difference $-25,95 \% \mathrm{Cl}-33$,17), whereas combination therapy with an aldosterone antagonist (ALDOA) plus ACEI or ARB reduced proteinuria by an additional $32 \%$ (mean difference $-32,95 \% \mathrm{Cl}-37,-27$ ). SBP after treatment with combination therapy vs. monotherapy was significantly lower with both the ACEI/ARB and ALDOA combinations. Dual therapy was associated with an increase in serum potassium, in particular with the ALDOA combination.
\end{abstract}

Limitations: Most studies were small and of short duration, and none included major patient outcome data such as kidney failure or death.

Conclusion: Dual RAS blockade in patients with DKD reduces proteinuria and SBP but increases the risk of hyperkalemia.

Keywords: Dual blockade; Diabetes; Angiotensin converting enzyme inhibitor; Angiotensin receptor blocker; Diabetic nephropathy; Diabetic kidney disease; Proteinuria

Diabetic kidney disease (DKD) is the leading cause of end-stage kidney disease (ESKD) in the United States [1,2]. Inhibitors of the renin-angiotensin system (RAS), such as angiotensin converting enzyme inhibitors (ACEIs) or angiotensin receptor blockers (ARBs), can slow the progression of DKD. However, despite these medications, the proportion of individuals with overt DKD (albuminuria $>300$ $\mathrm{mg} /$ day or $300 \mathrm{mg} / \mathrm{g}$ creatinine) who progress to ESKD remains high, with resultant high morbidity, mortality, and hospitalization rates [3]. Given the associated enormous human and financial costs of kidney failure, therapies to prevent the progression of kidney disease are vitally important.

Because of the beneficial effects of RAS inhibitors, there has been much interest in combination RAS inhibitor therapy in patients with DKD. However, this approach could increase risk, as individuals with DKD have an increased susceptibility to hyperkalemia [4]. A recent study in predominantly non-diabetic patients, ONTARGET (Ongoing Telmisartan Alone and in Combination with Ramipril Global End Point Trial), has cast doubt on the efficacy and safety of combined therapy [5]. However, in that study, the subset of patients with overt DKD appeared to fare somewhat but not significantly better with combination therapy. The ongoing Veterans Affairs Cooperative Study
VA NEPHRON-D (Combination Angiotensin Receptor Blocker and Angiotensin-Converting Enzyme Inhibitor for Treatment of Diabetic Nephropathy) [6] which is being carried out specifically in patients with overt DKD, will hopefully provide further risk/benefit information, though data from this study will not be available for several years.

The purpose of this meta-analysis was to determine the relative benefits [proteinuria and systolic blood pressure (SBP) reduction] and risks (hyperkalemia) as well as effects on glomerular filtration rate (GFR) of combined therapy vs. monotherapy in patients with overt DKD. Therefore, we restricted our analysis to randomized controlled trials of patients with overt proteinuria (studies of patients with microalbuminuria were not included). Since there are now

*Corresponding author: David J. Leehey, 111-L, Edward Hines Jr.VA Hospital, Hines, IL 60141, USA, Tel: 708-2022589; Fax: 708-2022256; E-mail: dleehey@lumc.edu

Received January 31, 2012; Accepted February 22, 2012; Published February 24, 2012

Citation: Pham JT, Schmitt BP, Leehey DJ (2012) Effects of Dual Blockade of the Renin-Angiotensin System in Diabetic Kidney Disease: A Systematic Review and Meta-Analysis. J Nephrol Therapeutic S2:003. doi:10.4172/2161-0959.S2-003

Copyright: (C) 2012 Pham JT, et al. This is an open-access article distributed under the terms of the Creative Commons Attribution License, which permits unrestricted use, distribution, and reproduction in any medium, provided the original author and source are credited. 
four different types of RAS blockers in clinical use [ACEIs, ARBs, aldosterone antagonists (ALDOAs), and direct renin inhibitors (DRIs)], studies using any dual combination therapy were included in this analysis providing there was a comparator monotherapy group. Both parallel-group and crossover trials were acceptable for inclusion.

\section{Methods}

\section{Data sources and searches}

We conducted searches of MEDLINE (Pubmed) (January 1966 to December 2011), EMBASE (January 1980 to December 2011) and CINAHL (January 1982 to December 2011) to identify all clinical trials published in English involving combination RAS blockers for treatment of DKD. Search terms used were "dual blockade OR combination therapy" AND "renin angiotensin system" AND "diabetes". In addition, we performed a manual search of the literature using the references of manuscripts and review articles. Finally, a search of the Cochrane database of systematic reviews was conducted. PRISMA guidelines were used for analysis and reporting in this manuscript [7].

\section{Study selection}

All randomized, controlled, parallel or crossover trials comparing combination RAS blockade to monotherapy for the treatment of DKD were considered for inclusion. Studies were subsequently included only if patients had overt albuminuria or proteinuria $(>300 \mathrm{mg} / 24 \mathrm{~h}$ or $>300 \mathrm{mg} / \mathrm{g}$ creatinine on a random specimen). Studies of patients with microalbuminuria were not included. We also excluded studies in which both diabetic and non-diabetic patients were enrolled if data were not given to analyze diabetic patients separately from nondiabetic patients. Two of the authors (J.T.P. and D.J.L.) independently identified potential articles, reviewed each abstract, and determined suitability for inclusion. Disagreements were resolved by consensus without a third party. The full publications of selected titles were then obtained and reviewed independently.

\section{Data extraction, quality assessment, and risk of bias}

A standardized data collection form was used to extract data from each study for important design characteristics and for quality assessment. The quality of each included study was assessed using the Jadad approach to quality review [8]. The Jadad score is calculated on a scale of $0-5$ by allotting one point for the following with five being the highest possible: randomization, valid method of randomization, double-blinded, matching placebos, and listing of drop-outs. In addition, risk of bias within studies was assessed by evaluating the methodological features of each study according to the Cochrane risk of bias assessment tool [9]. This tool takes into account random sequence generation, allocation concealment, blinding of participants and personnel, blinding of outcome assessment, incomplete outcome data, and selective reporting. To assess publication bias, a funnel plot was performed [10].

\section{Data synthesis and analysis}

The primary outcome analyzed was the mean difference in percent reduction in proteinuria (either albuminuria or total proteinuria) between the combination therapy and monotherapy groups. In most studies, proteinuria outcome data were presented as the geometric mean and $95 \%$ confidence interval (CI). Consequently, when working with log-transformed data we used the methods of Higgins et al. [11] In studies which had two monotherapy comparator arms (ACEI or ARB), the average of the means and standard deviations of the two arms was utilized. Secondary outcomes included change in SBP, change in GFR, change in serum potassium, and incidence of hyperkalemia. Statistical heterogeneity was assessed by the $\mathrm{I}^{2}$ test, with results of low, moderate, and high heterogeneity represented to be $25 \%, 50 \%$, and $75 \%$ respectively. Separate analyses were conducted in the following study subsets: combination ACEI/ARB studies, studies utilizing an ALDOA in combination with an ACEI or ARB, studies with the highest Jadad score of 5, and studies with low risk of bias based on the Cochrane tool. Forest plots were created with Review Manager software (version 5.0) using the random-effects model [10]. Although we analyzed the data using both a fixed-effects and random-effects assumption, results of the random-effects model (DerSimonian-Laird method) are reported because the analyses were similar.

\section{Results}

The result of the literature search is given in Figure 1. Sixteen articles were included in at least one analysis, with 12 articles suitable for primary outcome analysis. Summary results and selected characteristics of each clinical trial including study quality are given in Table 1. The risk of bias using the Cochrane assessment tool is shown in Table 2. Trial quality was variable, with Jadad scores varying from 1-5. Risk of bias was generally lower in studies with the highest Jadad scores. A funnel plot (Figure 2) suggests a low likelihood of publication bias.

\section{Efficacy outcomes: proteinuria}

Percent reduction in proteinuria: primary outcome: Twelve studies including 1067 patients were identified that met inclusion criteria and were analyzable for the primary outcome [12-23] (Table 1 , studies included in the primary outcome analysis identified by an asterisk). Ten of the studies were crossover-design trials, and two were parallel-design trials. Renal function was variable but no study included patients with severe renal dysfunction (GFR $<20 \mathrm{~mL} / \mathrm{min}$ ). Four studies included only type 1 diabetic patients; the other studies were either in type 2 diabetic patients (6 studies) or either type 1 or type 2 ( 2 studies). In 7 studies, combination ACEI/ARB therapy was compared to monotherapy; in 4 studies, combination therapy with the ALDOA spironolactone plus either an ACEI or ARB was compared to monotherapy with ACEI or ARB, and in one study the DRI aliskiren plus ARB was compared to ARB alone.

Overall, there was an additional 27\% (mean difference -27; 95\% CI $-32,-22$ ) relative reduction of proteinuria with combination therapy versus monotherapy (Figure 3a). When only the highest quality studies (Jadad score of 5) were examined [15-21], dual therapy resulted in a $29 \%$ (mean difference $-29 ; 95 \%$ CI $-33,-25$ ) additional reduction in proteinuria. When only studies with the lowest overall risk of bias were examined [12-21], nearly identical results were obtained (mean difference -30; 95\% CI -33, -26) (Figures not shown). $\mathrm{I}^{2}$ was $51 \%$, indicating moderate heterogeneity among studies.

Analysis by drug types: Due to the heterogeneity observed in primary outcome when all studies were pooled, subsequent analyses were performed separately for each drug type combination. Combination therapy with an ACEI plus ARB reduced proteinuria by an additional $25 \%$ (mean difference $-25,95 \%$ CI $-33,-17$ ), whereas combination therapy with an ALDOA plus ACEI or ARB reduced proteinuria, by an additional 32\% (mean difference -32, 95\% CI -37,27) (Figures $3 \mathrm{~b}$ and $3 \mathrm{c}$ ). There was only one study utilizing combination therapy with a DRI plus ARB, which resulted in a $20 \%$ reduction in 


\begin{tabular}{|c|c|c|c|c|c|c|c|c|c|c|}
\hline Author & $\begin{array}{l}\text { DM } \\
\text { Type }\end{array}$ & Proteinuria and/or GFR at baseline & Treatment/Design & $\begin{array}{l}\text { Treatment Arms (daily } \\
\text { dose in } \mathrm{mg} \text { ) }\end{array}$ & Control & $\mathrm{N}$ & $\begin{array}{l}\text { Drop out/ } \\
\text { Lost to } \\
\text { follow-up }\end{array}$ & $\begin{array}{c}\mathrm{N} \\
\text { analyzed }\end{array}$ & $\begin{array}{l}\text { Study } \\
\text { length }\end{array}$ & $\begin{array}{l}\text { Jadad } \\
\text { score } \\
(\max 5)\end{array}$ \\
\hline $\begin{array}{l}\text { Jacobsen } 2002 \\
(12)^{*}\end{array}$ & 1 & $\begin{array}{l}\text { AER }>300 \mathrm{mg} / 24 \mathrm{~h} \\
\mathrm{GFR} \geq 20 \mathrm{~mL} / \mathrm{min}\end{array}$ & ACEI + ARB/RCCT & $\begin{array}{c}\mathrm{C} 100 \text { or } \mathrm{E} / \mathrm{L} 20+\mathrm{I} 300 \mathrm{v} . \\
\mathrm{C} 100 \text { or } \mathrm{E} / \mathrm{L} 20\end{array}$ & Plac & 21 & 2 & 19 & 8 wks & 4 \\
\hline $\begin{array}{l}\text { Jacobsen } 2003 \\
\qquad(13)^{*}\end{array}$ & 1 & $\begin{array}{l}\text { AER }>300 \mathrm{mg} / 24 \mathrm{~h} \\
\mathrm{GFR} \geq 30 \mathrm{~mL} / \mathrm{min}\end{array}$ & ACEI + ARB/RCCT & $\mathrm{B} 20+\mathrm{V} 80 \mathrm{v} . \mathrm{B} 20$ or $\mathrm{V} 80$ & Plac & 20 & 2 & 18 & 8 wks & 4 \\
\hline $\begin{array}{l}\text { Jacobsen } 2003 \\
\quad(14)^{*}\end{array}$ & 1 & $\begin{array}{l}\text { AER }>300 \mathrm{mg} / 24 \mathrm{~h} \\
\mathrm{GFR} \geq 30 \mathrm{~mL} / \mathrm{min}\end{array}$ & ACEI + ARB/RCCT & $E 40+1300$ v. E40 & Plac & 24 & 0 & 24 & 8 wks & 4 \\
\hline $\begin{array}{l}\text { Mehdi } 2009 \\
(15)^{*}\end{array}$ & $1 / 2$ & $\begin{array}{c}\mathrm{ACR} \geq 300 \mathrm{mg} / \mathrm{g} \\
\mathrm{Cr} \leq 3.0 \text { (females) } 4.0 \text { (males) }\end{array}$ & $\begin{array}{l}\text { ACEI + ARB v. ACEI + } \\
\text { ALDOA/RCT }\end{array}$ & L80 + Los100 or Sp25 & Plac & 80 & 21 & 59 & 48 wks & 5 \\
\hline $\begin{array}{l}\text { Parving } 2008 \\
\qquad(16)^{*}\end{array}$ & 2 & $\begin{array}{l}\text { ACR }>300 \mathrm{mg} / \mathrm{g} \text { (or } 200 \mathrm{mg} / \mathrm{g} \text { if pt } \\
\text { taking RAS inhibitors) and }<3500 \mathrm{mg} / \mathrm{g} \\
\text { eGFR } \geq 30 \mathrm{~mL} / \mathrm{min} / 1.73 \mathrm{~m} 2\end{array}$ & $\mathrm{DRI}+\mathrm{ARB} / \mathrm{RCT}$ & $\begin{array}{c}\text { A150-300 + Los100 v. } \\
\text { Los } 100\end{array}$ & Plac & 599 & 75 & 524 & $6 \mathrm{mo}$ & 5 \\
\hline $\begin{array}{l}\text { Rossing } 2002 \\
(17)^{\star}\end{array}$ & 2 & $\begin{array}{c}\mathrm{AER}>300-1000 \mathrm{mg} / 24 \mathrm{~h} \\
\text { GFR } \geq 25 \mathrm{~mL} / \mathrm{min}\end{array}$ & ACEI + ARB/RCCT & $\begin{array}{l}\text { C100 or E/L20 + Cs8 v. } \\
\text { C100 or E/L20 }\end{array}$ & Plac & 18 & 1 & 17 & 8 wks & 5 \\
\hline $\begin{array}{l}\text { Rossing } 2003 \\
(18)^{*}\end{array}$ & 2 & $\begin{array}{l}\text { AER }>300-1000 \mathrm{mg} / 24 \mathrm{~h} \\
\quad \text { GFR } \geq 25 \mathrm{~mL} / \mathrm{min}\end{array}$ & ACEI + ARB/RCCT & $\begin{array}{c}C 150 \text { or } E / L 40+C s 16 v . \\
C 150 \text { or } E / L 40\end{array}$ & Plac & 20 & 0 & 20 & 8 wks & 5 \\
\hline $\begin{array}{l}\text { Rossing } 2005 \\
\qquad(19)^{\star}\end{array}$ & 2 & $\begin{array}{c}\text { AER }>300 \mathrm{mg} / 24 \mathrm{~h} \\
\mathrm{GFR} \geq 30 \mathrm{~mL} / \mathrm{min} / 1.73 \mathrm{~m} 2\end{array}$ & $\begin{array}{c}\text { ACEl or ARB + ALDOA/ } \\
\text { RCCT }\end{array}$ & $\begin{array}{c}\text { ACEl and/or ARB } \\
\text { (various) + Sp25 v. ACEI } \\
\text { or ARB }\end{array}$ & Plac & 21 & 1 & 20 & 8 wks & 5 \\
\hline $\begin{array}{l}\text { Schjoedt } 2005 \\
(20)^{*}\end{array}$ & 1 & $\begin{array}{c}\text { AER }>300 \mathrm{mg} / 24 \mathrm{~h} \\
\mathrm{GFR} \geq 30 \mathrm{~mL} / \mathrm{min} / 1.73 \mathrm{~m} 2\end{array}$ & $\begin{array}{c}\text { ACEl or ARB + ALDOA/ } \\
\text { RCCT }\end{array}$ & $\begin{array}{c}\text { ACEl and/or ARB } \\
\text { (various) + Sp25 v. ACEI } \\
\text { or ARB }\end{array}$ & Plac & 22 & 2 & 20 & $2 \mathrm{mo}$ & 5 \\
\hline $\begin{array}{l}\text { Schjoedt } 2006 \\
(21)^{*}\end{array}$ & $1 / 2$ & $\begin{array}{c}\text { AER }>2500 \mathrm{mg} / 24 \mathrm{~h} \\
\mathrm{GFR} \geq 30 \mathrm{~mL} / \mathrm{min} / 1.73 \mathrm{~m} 2\end{array}$ & $\begin{array}{c}\text { ACEI or ARB + ALDOA/ } \\
\text { RCCT }\end{array}$ & $\begin{array}{c}\text { ACEl and/or ARB various } \\
+ \text { Sp25 v. ACEI or ARB }\end{array}$ & Plac & 20 & 0 & 20 & $2 \mathrm{mo}$ & 5 \\
\hline Song $2003(22)^{*}$ & 2 & $\begin{array}{c}\mathrm{PER}>1 \mathrm{~g} / 24 \mathrm{~h} \\
\mathrm{CCr} 25-90 \mathrm{~mL} / \mathrm{min}\end{array}$ & $\begin{array}{l}\mathrm{ACEI}+\mathrm{ARB} \\
\mathrm{RCCT}\end{array}$ & R5-7.5 + Cs4-8 v. R5-7.5 & Plac & $\begin{array}{l}20 \\
\text { (of } 32 \\
\text { total) }\end{array}$ & 2 & 18 & 16 wks & 4 \\
\hline $\begin{array}{c}\text { Tan } 2010 \\
(23)^{*}\end{array}$ & 2 & AER > $300 \mathrm{mg} / 24 \mathrm{~h}$ & $\begin{array}{l}\text { ACEI + ARB } \\
\text { RCCT }\end{array}$ & $\begin{array}{l}\text { E20 + Los } 100 \text { v. E10 + } \\
\text { Los } 50 \text { v. E20 v. Los } 100\end{array}$ & No & 36 & 2 & 34 & 8 wks & 2 \\
\hline $\begin{array}{l}\text { Cetinkaya } 2004 \\
\quad(24)\end{array}$ & ? & $\mathrm{PER}>300 \mathrm{mg} / 24 \mathrm{~h}$ & $\begin{array}{l}\text { ACEI + ARB } \\
\text { RCCT }\end{array}$ & $\begin{array}{c}\text { E10 + Los50 v. E20 or } \\
\text { Los } 100\end{array}$ & No & 22 & 0 & 22 & 12 wks & 1 \\
\hline $\begin{array}{l}\operatorname{Kim} 2003 \\
(25)\end{array}$ & 2 & $\begin{array}{c}\text { PER }>1 \mathrm{~g} / 24 \mathrm{~h} \\
\mathrm{CCr} 25-90 \mathrm{~mL} / \mathrm{min} / 1.73 \mathrm{~m} 2\end{array}$ & $\begin{array}{l}\text { ACEI + ARB } \\
\text { RCCT }\end{array}$ & $\begin{array}{l}\text { R5.7 (R5 or } 7.5,5.7 \\
\text { average dose) + Cs4 } \\
\text { v. ACEI }\end{array}$ & Plac & $\begin{array}{c}24 \text { (of } 43 \\
\text { total) }\end{array}$ & 2 & 22 & 12 wks & 3 \\
\hline Matos 2005 (26) & 2 & $\begin{array}{c}\text { PER } 0.5-3 \mathrm{~g} / 24 \mathrm{~h} \\
\mathrm{CCr} \geq 40 \mathrm{~mL} / \mathrm{min} / 1.73 \mathrm{~m} 2\end{array}$ & $\begin{array}{l}\text { ACEI + ARB } \\
\text { RCCT }\end{array}$ & $P 8+1300$ v. P8 v. 1300 & No & 20 & 5 & 15 & 16 wks & 2 \\
\hline Song 2006 (27) & 2 & $\begin{array}{c}\text { PER }>1 \mathrm{~g} / 24 \mathrm{~h} \\
\mathrm{CCr} 30-59 \mathrm{~mL} / \mathrm{min} / 1.73 \mathrm{~m} 2\end{array}$ & $\begin{array}{l}\text { ACEI + ARB } \\
\text { RCCT }\end{array}$ & $R 5+C s 8$ v. R10 v. Cs16 & No & 25 & 4 & 21 & 16 wks & 3 \\
\hline
\end{tabular}

${ }^{*}$ Included in analysis for primary outcome; A, aliskiren; ACEI, angiotensin converting enzyme inhibitor; ACR, urinary albumin/creatinine ratio; AER, urinary albumin excretion rate; ALDOA aldosterone antagonist; ARB, angiotensin receptor blocker; C, captopril; Cs, candesartan; CCr, creatinine clearance; DM, diabetes mellitus; DRI, direct renin inhibitor; E, enalapril; eGFR, estimated glomerular filtration rate; GFR, glomerular filtration rate; I, irbesartan; L, lisinopril; Los, losartan; P, perindopril; PER, protein excretion rate; Plac, placebo; R, ramipril; RCCT, randomized controlled crossover trial; RCT, randomized controlled (parallel group) trial; Sp, spironolactone; $\mathrm{V}$, valsartan.

Table 1: Summary results and selected characteristics of each clinical trial.

\begin{tabular}{|c|c|c|c|c|c|c|}
\hline Author (ref) & $\begin{array}{l}\text { Random Sequence } \\
\text { Generation }\end{array}$ & $\begin{array}{c}\text { Allocation } \\
\text { Concealment }\end{array}$ & $\begin{array}{c}\text { Blinding of Participants } \\
\text { and Personnel }\end{array}$ & $\begin{array}{c}\text { Blinding of Outcome } \\
\text { Assessment }\end{array}$ & $\begin{array}{c}\text { Incomplete Outcome } \\
\text { Data }\end{array}$ & $\begin{array}{l}\text { Selective } \\
\text { Reporting }\end{array}$ \\
\hline Jacobsen $2002(12)^{*}$ & Low $^{1}$ & Low $^{1}$ & Unclear $^{3}$ & Low $^{6}$ & Low $^{7}$ & Unclear $^{9}$ \\
\hline Jacobsen $2003(13)^{*}$ & Low & Low & Unclear & Low & Low & Unclear \\
\hline Jacobsen $2003(14)^{*}$ & Low & Low & Unclear & Low & Low & Unclear \\
\hline Mehdi $2009(15)^{*}$ & Unclear $^{2}$ & Low & Low $^{4}$ & Low & Low & Unclear \\
\hline Parving $2008(16)^{\star}$ & Low & Low & Low & Low & Low & Unclear \\
\hline Rossing $2002(17)^{\star}$ & Low & Low & Low & Low & Low & Unclear \\
\hline Rossing $2003(18)^{*}$ & Low & Low & Low & Low & Low & Unlcear \\
\hline Rossing 2005 (19)* & Low & Low & Low & Low & Low & Unclear \\
\hline Schjoedt $2005(20)^{*}$ & Low & Low & Low & Low & Low & Unclear \\
\hline Schjoedt $2006(21)^{*}$ & Low & Low & Low & Low & Low & Unclear \\
\hline Song $2003(22)^{\star}$ & Unclear & Unclear $^{2}$ & Unclear & Low & Low & Unclear \\
\hline $\operatorname{Tan} 2010(23)^{\star}$ & Unclear & Unclear & $\mathrm{High}^{5}$ & Low & Low & Unclear \\
\hline Cetinkaya 2004 (24) & Unclear & Unclear & High & Low & Unclear $^{8}$ & $\mathrm{High}^{10}$ \\
\hline Kim 2003 (25) & Unclear & Unclear & Unclear & Low & Low & High \\
\hline Matos 2005 (26) & Low & Unclear & High & Low & Low & High \\
\hline Song 2006 (27) & Low & Unclear & High & Low & Low & High \\
\hline
\end{tabular}

Included in analysis for primary outcome; ${ }^{1}$ Method stated and appropriate; ${ }^{2}$ Method not stated; ${ }^{3}$ Characteristic of placebo not stated; ${ }^{4}$ Matching placebo; ${ }^{5}$ No placebo control; ${ }^{6}$ Not likely to be influenced by lack of blinding, even if present; ${ }^{7}$ Dropouts accounted for appropriately; ${ }^{8}$ Unclear if dropouts occurred; ${ }^{9}$ Insufficient information to judge; ${ }^{10} \mathrm{Primary}$ outcome unable to be included in meta-analysis 
proteinuria (mean difference $-20,95 \%$ CI -30, -10) compared to ARB monotherapy [16].

We found no effect of baseline proteinuria on the primary outcome with either drug combination. There was a significant antiproteinuric effect of dual blockade with ACEI plus ARB in patients with $<1 \mathrm{~g}$ daily proteinuria at baseline (mean difference -29; $95 \%$ CI $-35,-23$ ) as well as in patients with $\geq 1 \mathrm{~g}$ daily proteinuria at baseline (mean difference $-24,95 \%$ CI $-42,-6)$. Similarly, there was a significantly greater antiproteinuric effect of dual blockade with an ALDOA plus ACEI or ARB both in patients with less proteinuria at baseline $(<1$ $\mathrm{g} / 24 \mathrm{~h}$ or $<1 \mathrm{~g} / \mathrm{g}$ creatinine) (mean difference $-30,95 \% \mathrm{CI}-42,-18$ ) and in patients with greater degrees of baseline proteinuria $(\geq 1 \mathrm{~g} / 24 \mathrm{~h}$ or $\geq 1 \mathrm{~g} / \mathrm{g}$ creatinine) (mean difference -33, 95\% CI -39, -26) (Figure not shown). In the one study using a DRI plus ARB, baseline albuminuria was $<1 \mathrm{~g} / \mathrm{g}[16]$.

Effect of combination therapy on SBP: There was a $3.5 \mathrm{mmHg}$ decrease (mean difference -3.5, 95\% CI -6.5, -0.6) in SBP with ACEI plus ARB vs. monotherapy (Figure $4 \mathrm{a}$ ), and $6.2 \mathrm{mmHg}$ decrease (mean

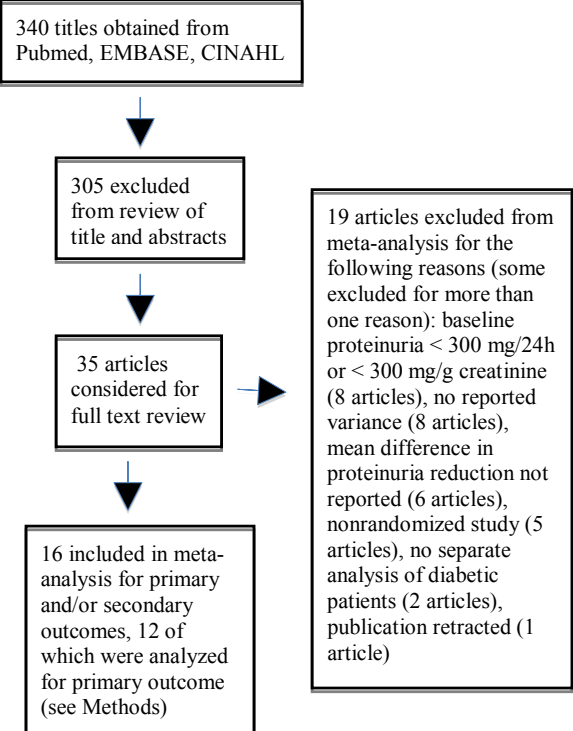

Figure 1: Identification and selection of studies included in this meta-analysis.

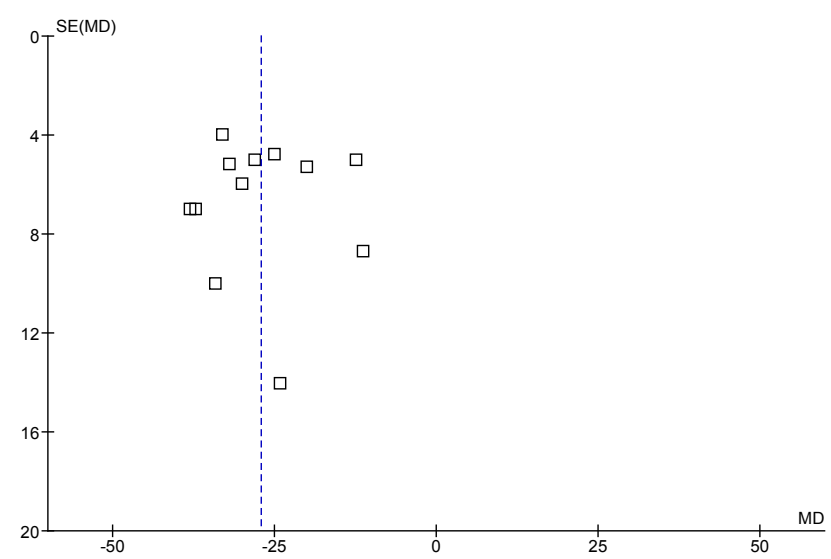

Figure 2: Funnel plot showing low likelihood of publication bias. difference $-6.2,95 \% \mathrm{CI}-11.6,-0.7)$ with ALDOA in combination with an ACEI or ARB vs. monotherapy (Figure 4b). There was a $2 \mathrm{mmHg}$ decrease in SBP with DRI plus ARB vs. ARB alone, which was not significant [16].

Effect of combination therapy on GFR: There was a slight but insignificantly greater decline in GFR with dual blockade relative to monotherapy with both the ACEI/ARB combination $(-0.6 \mathrm{~mL} / \mathrm{min}$; 95\% CI -7.6, 6.4), and with dual blockade containing an ALDOA (-1.0 $\mathrm{mL} / \mathrm{min}$; 95\% CI -6.1, 4.1) (Figure not shown). There was a slightly but not significant decrease in GFR $(-1.4 \mathrm{~mL} / \mathrm{min})$ with a DRI plus ARB vs. ARB alone [16]

\section{Safety outcomes: change in serum potassium and incidence of hyperkalemia}

Serum potassium was slightly but not significantly higher with the ACEI/ARB combination (mean difference 0.09 meq/L; 95\% CI -0.01, 0.20 ). On the other hand, it was significantly higher with the ALDOA combination (mean difference $0.23 \mathrm{meq} / \mathrm{L} ; 95 \%$ CI 0.07, 0.39) (Figures $5 a$ and $5 b)$.

In some studies, the number of hyperkalemic episodes was reported. ACEI/ARB combination therapy was associated with a 2.16fold (0.70-6.67) increase in risk (Figure 5c) vs. a 2.99-fold (0.86-10.38) increase in studies using an ALDOA combination (Figure $5 \mathrm{~d}$ ). In the one study using DRI plus ARB, there was a 2.77-fold (1.01-7.60) increase in risk. In all but 5 instances, hyperkalemia was clinically significant (i.e. serum potassium was $>6.0 \mathrm{meq} / \mathrm{L}$, the patient required treatment for hyperkalemia, and/or the patient was dropped from the study due to hyperkalemia).

\section{Discussion}

Dual blockade of the RAS has been proposed to further improve clinical outcomes in patients with DKD. Higher levels of proteinuria are associated with a faster rate of decline in renal function in DKD $[28,29]$ and reduction in proteinuria and BP by ACEIs or ARBs is associated with decreased risk of progression [30,31,32] Thus it is reasonable to predict that a further reduction in proteinuria and BP by dual RAS blockade might further decrease progression. In this metaanalysis, we found that combination RAS inhibitor therapy was indeed more effective than monotherapy in reducing proteinuria in DKD, and this occurred both when baseline proteinuria was $<1$ gram/day as well as when it was $\geq 1$ gram/day at baseline. Dual RAS blockade was also associated with a greater decline in SBP. On the other hand, inhibition of the RAS in patients with DKD predisposes to hyperkalemia [33]. Combination RAS inhibition would be expected to further increase this risk, especially in patients with impaired GFR. In our meta-analysis, although the overall increment in serum potassium was relatively low $(\sim 0.1-0.2 \mathrm{meq} / \mathrm{L})$, a substantial number of patients ( $\sim 5 \%$ in ACEI/ ARB and $\sim 23 \%$ in ALDOA groups) developed clinically significant hyperkalemia. In addition to the well-known adverse and potentially fatal cardiac risk of severe hyperkalemia, even modest degrees of hyperkalemia appear to offset the renoprotective effects of RAS blockade [34].

Most studies investigating dual RAS blockade in various renal diseases have focused on combination ACEI/ARB therapy [1214,17,18,22-27]. Both ACEIs and ARBs suppress aldosterone secretion; however, with prolonged treatment, aldosterone levels increase, a phenomenon termed "aldosterone escape" [35]. Moreover, there is a secondary increase in renin with either ACEI or ARB therapy. It has 


\begin{tabular}{|c|c|c|c|c|}
\hline Study or Subgroup & Mean Difference & SE & Weight & $\begin{array}{l}\text { Mean Difference } \\
\text { IV, Random, } 95 \% \text { C }\end{array}$ \\
\hline Jacobsen 2002 (12) & -37 & 7 & $7.4 \%$ & $-37.00[-50.72,-23.28]$ \\
\hline Jacobsen 2003 JASN (13) & -38 & 7 & $7.4 \%$ & $-38.00[-51.72,-24.28]$ \\
\hline Jacobsen $2003 \mathrm{KI}(14)$ & -25 & 4.8 & $10.7 \%$ & $-25.00[-34.41,-15.59]$ \\
\hline Mehdi ACE+AA (15) & -34 & 10 & $4.6 \%$ & $-34.00[-53.60,-14.40]$ \\
\hline Parving 2008 (16) & -20 & 5.3 & $9.9 \%$ & $-20.00[-30.39,-9.61]$ \\
\hline Rossing 2002 (17) & -24 & 14 & $2.7 \%$ & $-24.00[-51.44,3.44]$ \\
\hline Rossing 2003 (18) & -28 & 5 & $10.4 \%$ & $-28.00[-37.80,-18.20]$ \\
\hline Rossing 2005 (19) & -33 & 4 & $12.2 \%$ & $-33.00[-40.84,-25.16]$ \\
\hline Schjoedt 2005 (20) & -30 & 6 & $8.8 \%$ & $-30.00[-41.76,-18.24]$ \\
\hline Schjoedt 2006 (21) & -32 & 5.2 & $10.0 \%$ & $-32.00[-42.19,-21.81]$ \\
\hline Song 2003 (22) & -12.4 & 5 & $10.4 \%$ & $-12.40[-22.20,-2.60]$ \\
\hline Tan $2010(23)$ & -11.2 & 8.7 & $5.6 \%$ & $-11.20[-28.25,5.85]$ \\
\hline Total $(95 \% \mathrm{Cl})$ & \multicolumn{4}{|r|}{$-27.09[-31.99,-22.19]$} \\
\hline Heterogeneity: $\mathrm{Tau}^{2}=35.4$ & $\mathrm{Chi}^{2}=22.25, \mathrm{df}=1$ & $11(P$ & $=0.02)$ & $2^{2}=51 \%$ \\
\hline
\end{tabular}

Test for overall effect: $Z=10.83(P<0.00001)$
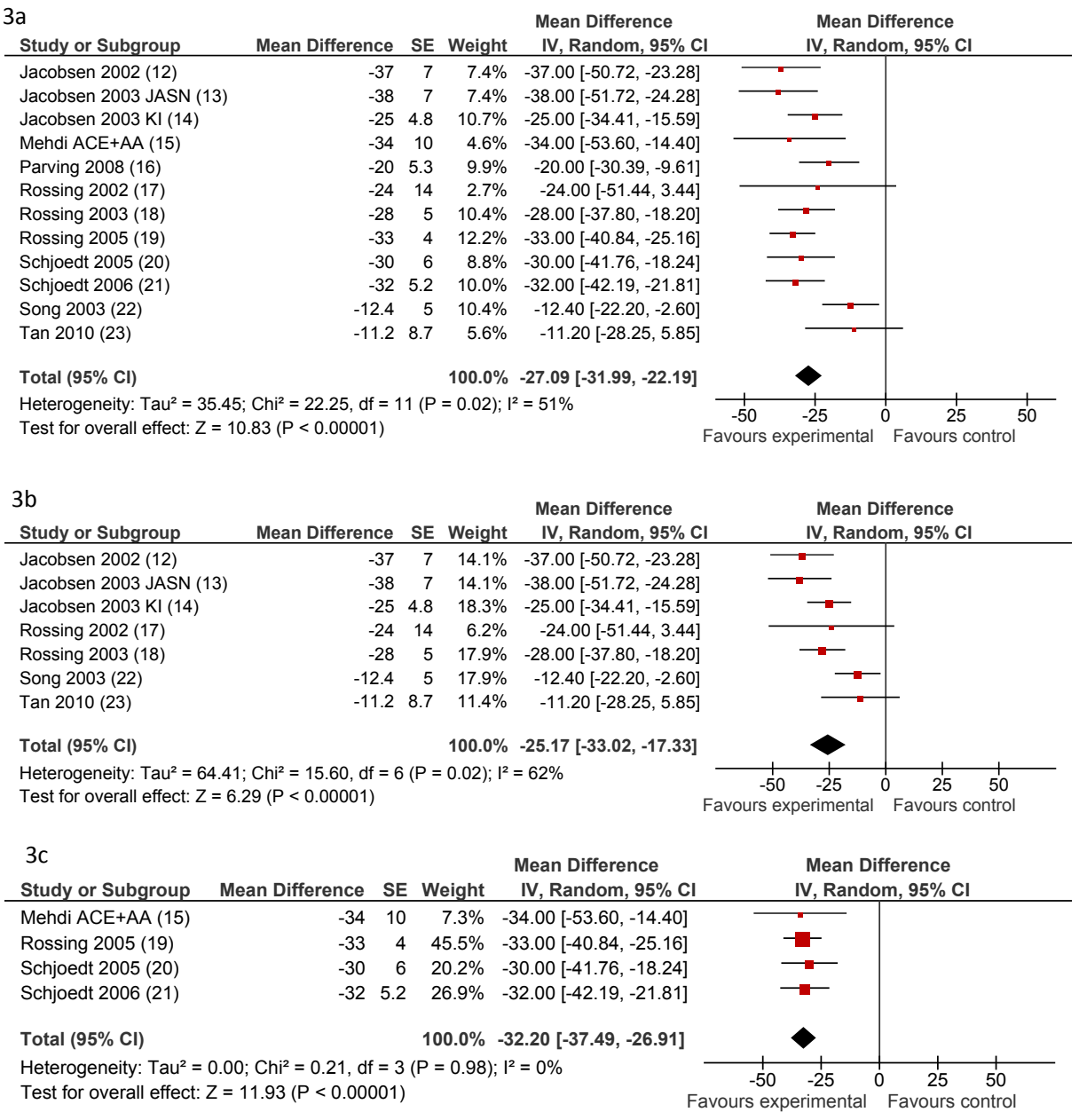

Figure 3: A) Percent reduction in proteinuria. Combination therapy resulted in an additional $27 \%$ relative reduction in proteinuria compared to monotherapy. B) Combination therapy with an ACEI and ARB vs. monotherapy led to further $25 \%$ reduction in proteinuria. C) Combination therapy with an ALDOA plus an ACEI or $A R B$ vs. ACEI or ARB alone led to $32 \%$ further reduction in proteinuria.

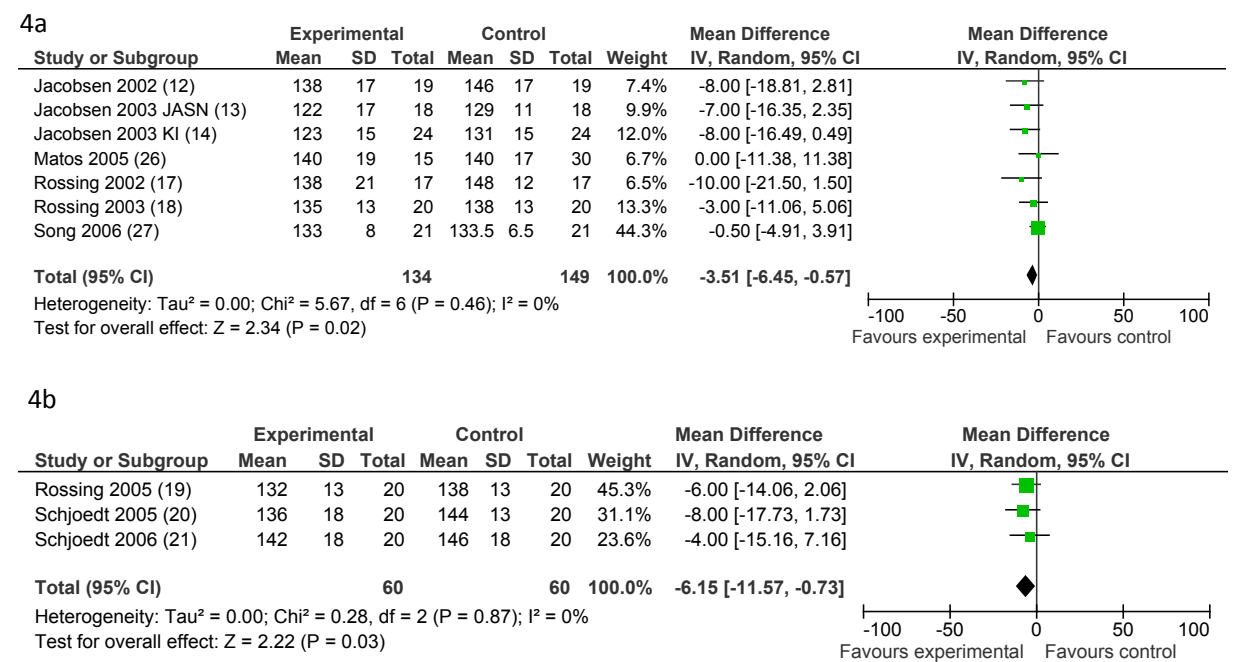

Figure 4: A) Difference in SBP after treatment with ACEI/ARB combination therapy vs. monotherapy. SBP was slightly (-3.5 mmHg) but significantly lower after combination therapy. B) Difference in SBP after treatment with ALDOA combination therapy vs. monotherapy. SBP was slightly (-6.2 mmHg) but significantly lower after combination therapy. 
thus been proposed that the addition of an ALDOA or DRI to ACEI or ARB will have additional renoprotective benefits in DKD [16]. However, although ALDOAs can reduce proteinuria, combination therapy including an ALDOA results in a substantial risk of hyperkalemia, especially in patients with more severe chronic kidney disease $[15,36]$. In the recent study of Mehdi et al. [15], in which some subjects had substantial renal dysfunction, serum potassium $>6 \mathrm{meq} / \mathrm{L}$ was noted in $52 \%$ of patients treated with combined high-dose ACEI plus low-dose (25 mg) spironolactone. In a large study of microalbuminuric patients with generally preserved renal function (thus not included in this analysis), the ALDOA eplerenone when added to the ACEI lisinopril decreased albuminuria by about $40 \%$ but $8 \%$ of patients treated with the higher dose of eplerenone had to be withdrawn from the study due to hyperkalemia [36]. Thus the relative benefit vs. risk of dual therapy including an ALDOA needs to be considered even in patients with less severe kidney disease. Moreover, the mechanism of proteinuria reduction with ALDOAs may not be due to aldosterone blockade, as addition of furosemide to either ACEI or ARB also decreases proteinuria [37]. The combination of the DRI aliskiren and an ARB decreased proteinuria more than $\mathrm{ARB}$ alone with a relatively low rate of hyperkalemia in one study [16]. However, a larger study comparing the combination of aliskiren and either an ACEI or ARB to an ACEI or ARB alone was terminated after 18-24 months in December 2011 due to an increased risk for non-fatal stroke, renal complications, hyperkalemia, and hypotension in patients taking aliskiren [38].

A)

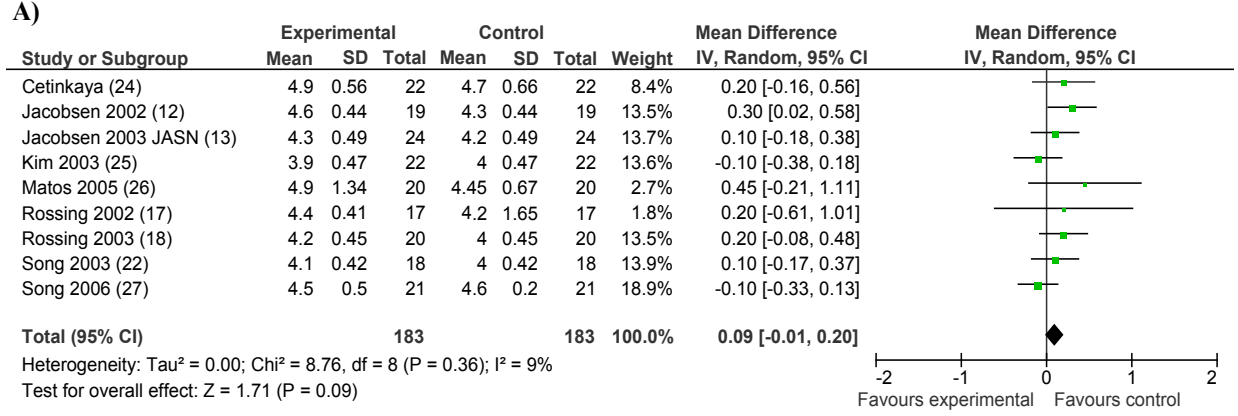

B)

\begin{tabular}{|c|c|c|c|c|c|c|c|}
\hline & \multicolumn{3}{|c|}{ Experimental } & \multicolumn{3}{|c|}{ Control } & \\
\hline Study or Subgroup & Mean & SD & Total & Mean & SD & Total & Weigh \\
\hline Rossing 2005 (19) & 4.3 & 0.45 & 20 & 4 & 0.45 & 20 & $32.3 \%$ \\
\hline Schjoedt 2005 (20) & 4.2 & 0.45 & 22 & 4 & 0.45 & 22 & $35.5 \%$ \\
\hline Schjoedt 2006 (21) & 4.3 & 0.45 & 20 & 4.1 & 0.45 & 20 & $32.3 \%$ \\
\hline Total $(95 \% \mathrm{Cl})$ & & & 62 & & & 62 & $100.0 \%$ \\
\hline
\end{tabular}

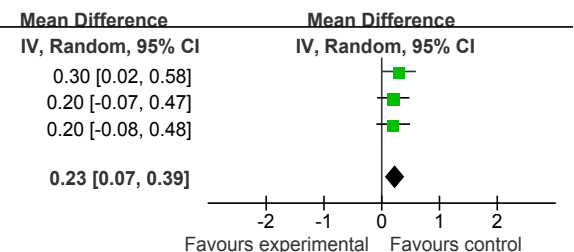

C)

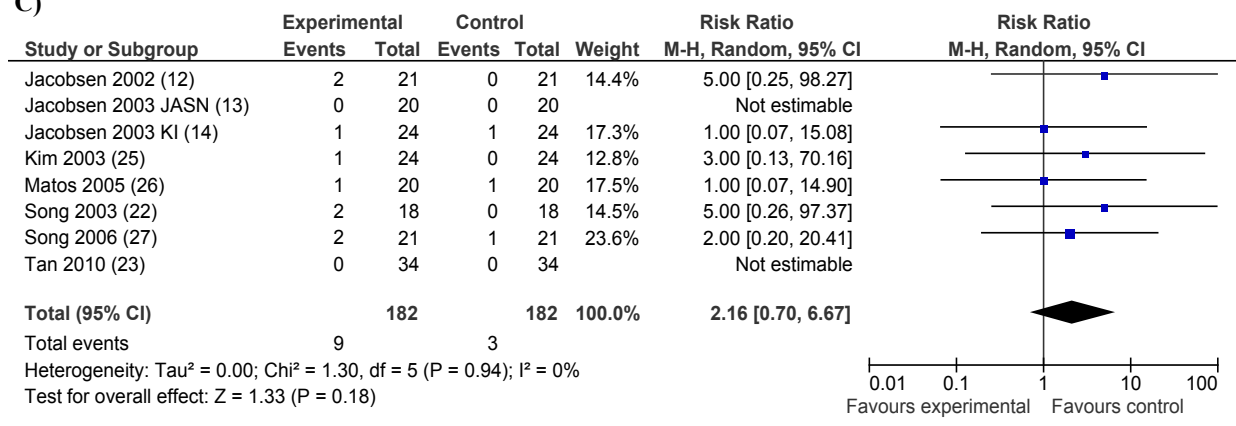

D)

\begin{tabular}{|c|c|c|c|c|c|}
\hline \multirow[b]{2}{*}{ Study or Subgroup } & \multicolumn{2}{|c|}{ Experimental } & \multicolumn{2}{|c|}{ Control } & \multirow[b]{2}{*}{ Weight } \\
\hline & Events & Total & Events & Total & \\
\hline Mehdi ACE+AA (15) & 14 & 27 & 2 & 27 & $48.8 \%$ \\
\hline Rossing 2005 (19) & 1 & 21 & 0 & 21 & $13.9 \%$ \\
\hline Schjoedt 2005 (20) & 1 & 22 & 0 & 22 & $13.9 \%$ \\
\hline Schjoedt 2006 (21) & 1 & 20 & 2 & 20 & $23.4 \%$ \\
\hline Total $(95 \% \mathrm{Cl})$ & & 90 & & 90 & $100.0 \%$ \\
\hline Total events & 17 & & 4 & & \\
\hline $\begin{array}{l}\text { Heterogeneity: } \mathrm{Tau}^{2} \\
\text { Test for overall effect }\end{array}$ & .33; Chi ${ }^{2}$ & $3.70, c$ & If $=3(P=$ & $=0.30)$ & $I^{2}=19 \%$ \\
\hline
\end{tabular}

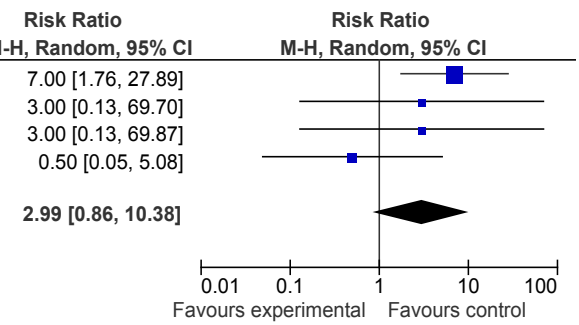

Figure 5: A) Difference in serum potassium after treatment with ACEI/ARB combination therapy vs. monotherapy. Serum potassium was slightly (0.09 mEq/L) but not significantly higher after combination therapy. B) Difference in serum potassium after treatment with ALDOA combination therapy vs. monotherapy. Serum potassium was slightly $(0.23 \mathrm{mEq} / \mathrm{L})$ but significantly higher after combination therapy. C) Risk of hyperkalemia after treatment with ACEI/ARB combination therapy vs. monotherapy. There was a 2.16 -fold increased risk of hyperkalemia with combination therapy. D) Risk of hyperkalemia after treatment with ALDOA combination therapy vs. monotherapy. There was a 2.99 -fold increase risk of hyperkalemia with combination therapy. 
The recent ONTARGET study has cast doubt on the efficacy and in particular safety of dual RAS blockade with ACEIs and ARBs [5]. However, it is difficult to apply the results of this study to patients with $\mathrm{DKD}$, as this study was in primarily non-diabetic patients, and only $4 \%$ of the diabetic patients in this trial had overt proteinuria. One of the major concerns of the ONTARGET trial was the increased need for acute dialysis in the combination treatment arm due to rapid worsening of renal function in some patients. It is well known that BP reduction, especially with RAS inhibitors, can lead to a decline in GFR during the first several months of therapy $[39,40]$. Assessing changes in GFR in short-term studies using RAS inhibitors is thus problematic. Interestingly, despite the fact that many studies were of short duration, we did not find differences in change in GFR between the control and intervention groups in this meta-analysis.

In a previous meta-analysis of combination RAS blockade for DKD, only ACEI plus ARB therapy was examined, the authors included nonrandomized studies and studies of microalbuminuric patients (e.g., without overt proteinuria), and only one study was longer than 12 weeks in duration [41]. Other meta-analyses examining combination therapy have combined together various etiologies of renal disease, thus including patients with and without DKD [42]. In contrast, we focused our study on patients with DKD and overt proteinuria and included only randomized studies. Another strength of our meta-analysis is the inclusion of studies involving various types of RAS blocker combinations, including some recent larger-scale studies which employed ALDOAs and DRIs which are now used in clinical practice.

However, our study does have limitations. Not all studies included were of high quality or free of bias, and the majority of the studies were small in sample size and of limited duration. However, restriction of the analysis to only higher quality studies or studies with low risk of bias did not appreciably change the findings. Another confounding factor could be differences in degree of renal impairment among studies. The absence of patient outcome data necessitated use of a surrogate marker (proteinuria) for the primary outcome. Furthermore, there was likely an underrepresentation of African and/or African-American patients.

\section{Summary and Conclusion}

In conclusion, dual RAS inhibition is an option to decrease proteinuria and control BP in patients with DKD but is associated with an increased risk of hyperkalemia. Reduction in proteinuria and BP are clearly surrogate outcomes, and no large scale randomized clinical trials assessing the effects of dual RAS blockade on clinical outcomes such as progression to ESKD, cardiovascular morbidity and mortality, and death have been completed. Two such trials, both using combination ACEI plus ARB therapy compared to monotherapy, the aforementioned NEPHRON-D [6] and the VALID trial (Preventing ESRD in Overt Nephropathy in Type 2 Diabetes) [43], are currently in progress. In our meta analysis, the increase in serum potassium was somewhat less with combined ACEI/ARB than with other combinations. We believe that, at this time, dual therapy should probably only be attempted with the ACEI/ARB combination and only in selected patients (e.g., those with macroalbuminuria and normal serum potassium levels on RAS blockade monotherapy).

\section{Acknowledgements}

The authors declare they have no financial conflicts of interest.

\section{References}

1. United States Renal Data System (USRDS) Annual Data Report (2010) Bethesda, MD: National Institutes of Health, National Institute of Diabetes and Digestive and Kidney Diseases

2. Burrows NR, Li Y, Geiss LS (2010) Incidence of treatment for end-stage renal disease among individuals with diabetes in the U.S. continues to decline. Diabetes Care 33: 73-77.

3. Collins AJ, Li S, Gilbertson DT, Liu J, Chen SC, et al. (2003) Chronic kidney disease and cardiovascular disease in the Medicare population. Kidney Int Suppl 87: 24-31.

4. Palmer BF (2004) Managing hyperkalemia caused by inhibitors of the reninangiotensin-aldosterone system. New Eng J Med 351: 585-592.

5. Mann JF, Schmieder RE, McQueen M, Dyal L, Schumacher H, et al. (2008) Renal outcomes with telmisartan, ramipril, or both, in people at high vascular risk (the ONTARGET study): a multicentre, randomised, double-blind, controlled trial. Lancet 372: 547-553.

6. Fried LF, Duckworth W, Zhang JH, O'Connor T, Brophy M, et al. (2009) Design of combination angiotensin receptor blocker and angiotensin-converting enzyme inhibitor for treatment of diabetic nephropathy (VA NEPHRON-D). Clin J Am Soc Nephrol 4: 361-368.

7. Liberati A, Altman DG, Tetzlaff J, Mulrow C, Gotzsche PC, et al. (2009) The PRISMA statement for reporting systematic reviews and meta-analyses of studies that evaluate health care interventions: explanation and elaboration. PLoS Medicine 6: 1000100

8. Jadad AR, MooreRA, Carroll D, Jenkinson C, Reynolds DJ, et al. (1996). Assessing the Quality of reports of Randomized Clinical Trials: Is Blinding Necessary?. Control Clin Trials 17: 1-12.

9. Higgins JPT, Altman DG (2011) Assessing risk of bias in included studies. In Higgins JPT, Green S (editors). Cochrane Handbook for Systematic Reviews of Interventions Version 5.1. The Cochrane Collaboration Chapter 8.

10. Review Manager (RevMan) [Computer program] (2008). Version 5.0 Copenhagen: The Nordic Cochrane Centre, The Cochrane Collaboration.

11. Higgins JP, White IR, Anzures-Cabrera J (2008) Meta-analysis of skewed data: Combining results reported on log-transformed or raw scales. Statist Med 27 6072-6092.

12. Jacobsen $P$, Andersen $S$, Rossing $K$, Hansen BV, Parving HH, et al. (2002) Dual blockade of the renin-angiotensin system in type 1 patients with diabetic nephropathy. Nephrol Dial Transplant 17: 1019-1024.

13. Jacobsen P, Andersen S, Jensen BR, Parving HH (2003) Additive effect of ACE inhibition and angiotensin II receptor blockade in type I diabetic patients with diabetic nephropathy. J Am Soc Nephrol 14: 992-999.

14. Jacobsen P, Andersen S, Rossing K, Jensen BR, Parving HH, et al. (2003) Dual blockade of the renin-angiotensin system versus maximal recommended dose of ACE inhibition in diabetic nephropathy. Kidney Int 63: 1874-1880.

15. Mehdi UF, Adams-Huet B, Raskin P, Vega GL, Toto RD, et al. (2009) Addition of angiotensin receptor blockade or mineralocorticoid antagonism to maximal angiotensin-converting enzyme inhibition in diabetic nephropathy. J Am Soc Nephrol 20: 2641-2650.

16. Parving HH, Persson F, Lewis JB, Lewis EJ, Hollenberg NK, et al. (2008) Aliskiren combined with losartan in type 2 diabetes and nephropathy. N Engl $J$ Med 358: 2433-2446.

17. Rossing K, Christensen PK, Jensen BR, Parving HH (2002) Dual blockade of the renin-angiotensin system in diabetic nephropathy: a randomized doubleblind crossover study. Diabetes Care 25: 95-100.

18. Rossing K, Jacobsen P, Pietraszek L, Parving HH. (2003) Renoprotective effects of adding angiotensin II receptor blocker to maximal recommended doses of ACE inhibitor in diabetic nephropathy: a randomized double-blind crossover trial. Diabetes Care 26: 2268-2274.

19. Rossing K, Schjoedt KJ, Smidt UM, Boomsma F, Parving HH, et al. (2005) Beneficial effects of adding spironolactone to recommended antihypertensive treatment in diabetic nephropathy: a randomized, double-masked, cross-over study. Diabetes Care 28: 2106-2112.

20. Schjoedt KJ, Rossing K, Juhl TR, Boomsma F, Rossing P, et al. (2005) Beneficial impact of spironolactone in diabetic nephropathy. Kidney Int 68 : 2829-2836. 
21. Schjoedt KJ, Rossing K, Juhl TR, Boomsma F, Tarnow L, et al. (2006) Beneficial impact of spironolactone on nephrotic range albuminuria in diabetic nephropathy. Kidney Int 70: 536-542.

22. Song JH, Lee SW, Suh JH, Kim ES, Hong SB, et al. (2003) The effects of dual blockade of the renin-angiotensin system on urinary protein and transforming growth factor-beta excretion in 2 groups of patients with $\lg \mathrm{A}$ and diabetic nephropathy. Clin Nephrol 60: 318-326.

23. Tan F, Mukherjee JJ, Lee KO, Lim P, Liew CF, et al. (2010) Dual blockade of the renin-angiotensin-aldosterone system is safe and effective in reducing albuminuria in Asian type 2 diabetic patients with nephropathy. Singapore Med J 51: 151-156

24. Cetinkaya R, Odabas AR, Selcuk Y (2004) Anti-proteinuric effects of combination therapy with enalapril and losartan in patients with nephropathy due to type 2 diabetes. Int J Clin Pract 58: 432-435.

25. Kim MJ, Song JH, Suh JH, Lee SW, Kim GA (2003). Additive antiproteinuric effect of combination therapy with ACE inhibitor and angiotensin II receptor antagonist: differential short-term response between IgA nephropathy and diabetic nephropathy. Yonsei Med J 44: 463-472.

26. Matos JP, de Lourdes Rodrigues M, Ismerim VL, Boasquevisque EM, Genelhu $\mathrm{V}$, et al. (2005) Effects of dual blockade of the renin angiotensin system in hypertensive type 2 diabetic patients with nephropathy. Clin Nephrol 64: 180189.

27. Song JH, Cha SH, Lee HJ, Lee SW, Park GH, et al. (2006) Effect of low-dose dual blockade of renin-angiotensin system on urinary TGF-beta in type 2 diabetic patients with advanced kidney disease. Nephrol Dial Transplant 21: $683-689$

28. Atkins RC, Briganti EM, Lewis JB, Hunsicker LG, Braden G, et al. (2005) Proteinuria reduction and progression to renal failure in patients with type 2 diabetes mellitus and overt nephropathy. Am J Kidney Dis 45: 281-287.

29. de Zeeuw D, Remuzzi G, Parving HH, Keane WF, Zhang Z, et al. (2004) Proteinuria, a target for renoprotection in patients with type 2 diabetic nephropathy: lessons from RENAAL. Kidney Int 65: 2309-2320.

30. Keane WF, Brenner BM, de Zeeuw D, Grunfeld JP, McGill J, et al. (2003) The risk of developing end-stage renal disease in patients with type 2 diabetes and nephropathy: the RENAAL study. Kidney Int 63: 1499-1507.

31. Bakris GL, Weir MR, Shanifar S, Zhang Z, Douglas J, et al. (2003) Effects of blood pressure level on progression of diabetic nephropathy: results from the RENAAL study. Arch Intern Med 163: 1555-1565.
32. Middleton JP, Lewis J, for the Collaborative Study Group (2002) Predictors of renal outcome in type 2 diabetic nephropathy [abstract]. J Am Soc Nephrol 13 249-250.

33. Lewis EJ, Hunsicker LG, Clarke WR, Berl T, Pohl MA, et al. (2001) Renoprotective effect of the angiotensin-receptor antagonist irbesartan in patients with nephropathy due to type 2 diabetes. New Eng J Med 345: 851 860 .

34. Miao Y, Dobre D, Heerspink HJ, Brenner BM, Cooper ME, et al. (2011) Increased serum potassium affects renal outcomes: a post hoc analysis of the Reduction of Endpoints in NIDDM with the Angiotensin II Antagonist Losartan (RENAAL) trial. Diabetologia 54: 44-50.

35. Sato A, Hayashi K, Naruse M, Saruta T (2003) Effectiveness of aldosterone blockade in patients with diabetic nephropathy. Hypertension 41: 64-68.

36. Epstein M, Williams GH, Weinberger M, Lewin A, Krause S, et al. (2006) Selective aldosterone blockade with eplerenone reduces albuminuria in patients with type 2 diabetes. Clin J Am Soc Nephrol 1: 940-951.

37. Esnault VL, Ekhlas A, Delcroix C, Moutel MG, Nguyen JM, et al. (2005) Diuretic and enhanced sodium restriction results in improved antiproteinuric response to RAS blocking agents. J Am Soc Nephrol 16: 474-481.

38. Pharmactopics Pharmaceutical News (2012) http://www.pharmatopics com/2011/12/novartis-terminates-hypertension-therapy-trial-altitude.

39. Bakris GL, Williams M, Dworkin L, Elliott WJ, Epstein M, et al. (2000) Preserving renal function in adults with hypertension and diabetes: A consensus approach. Am J Kidney Dis 36: 646-661.

40. Lewis J, Greene T, Appel L, Contreras G, Douglas J, et al. (2004) A comparison of iothalamate-GFR and serum creatinine-based outcomes: acceleration in the rate of GFR decline in the African American Study of Kidney Disease and Hypertension. J Am Soc Nephrol 15: 3175-3183.

41. Jennings DL, Kalus JS, Coleman CI, Manierski CJ (2007) Combination therapy with an ACE inhibitor and an angiotensin receptor blocker for diabetic nephropathy: a meta-analysis. Diabet Med 24: 486-493.

42. Kunz R, Friedrich C, Wolbers M, Mann JF (2008) Meta-analysis: effect of monotherapy and combination therapy with inhibitors of the renin angiotensin system on proteinuria in renal disease. Ann Intern Med 148: 30-48.

43. Clinical Trials at Villa Camozzi VALID trial (2012) http://clintrials.marionegri.it/ index.php/main-trials/main-trials-ongoing/70.html. 